\title{
The Effect of Sensorimotor Replacement on Smoking Cessation and Craving
}

\author{
Dunja Przulj ${ }^{*}$, Hayden McRobbie and Peter Hajek
}

\author{
Tobacco Dependence Research Unit, Wolfson Institute of Preventive Medicine, Queen Mary University of London, UK
}

\begin{abstract}
Current treatments for smoking cessation such as nicotine replacement therapy or varenicline address the primary reinforcer of smoking (nicotine), but sensorimotor stimuli (e.g. smell/taste of smoke, inhaling/exhaling, airway sensations, holding the cigarette) may act as secondary reinforcers and also contribute to smoking reward. Addressing both these aspects of smoking may help to enhance smoking cessation treatment. The aim of this review was to examine whether sensorimotor replacement can help to alleviate craving and aid smoking cessation. Three sensorimotor replacement products were examined: non-nicotine inhalators/aerosols, de-nicotinised cigarettes and electronic cigarettes. The current research suggests that sensorimotor replacement may enhance the efficacy of nicotine replacement therapy, but is unlikely to be useful if used alone. Electronic cigarettes may be the most promising approach, due to the combination of nicotine delivery and sensorimotor input.
\end{abstract}

Keywords: Craving, de-nicotinised cigarettes, electronic cigarettes, inhalator, nicotine, sensorimotor replacement, smoking cessation.

\section{INTRODUCTION}

Nicotine has long been identified as the fundamental component in tobacco addiction. Recognising the addictive nature of nicotine has led to the development of effective treatments for smoking cessation such as nicotine replacement therapy (NRT) and varenicline. Although medication can enhance people's chances of successfully quitting [1], long-term cessation rates are overall low. This suggests there may be more to smoking and tobacco addiction than just nicotine.

It could be argued that the efficacy of NRT is limited because it is underused and/or does not deliver nicotine in large enough doses or quickly enough in comparison to cigarettes. Although it is likely that these are contributing factors, it has also been proposed that sensorimotor factors such as holding the cigarette, inhaling/exhaling, smell/taste of smoke, airway sensations, play a role in tobacco addiction, which current treatments do not fully address [2]. Sensorimotor stimuli may contribute to smoking reward through their association with the pharmacological effects of nicotine [3]. This happens through classical or 'Pavlovian' conditioning, whereby a previously neutral stimulus becomes rewarding if it is closely followed by a real reward and may eventually acquire an independent incentive value. There is some evidence that such factors can play a role in the rewarding effects of smoking. Blocking the sensations of cigarette smoke by anesthetising the upper and lower respiratory tract led to less enjoyment of smoking [4]. Nicotine administered intravenously was perceived as less

*Address correspondence to this author at the Tobacco Dependence Research Unit, Bart's and the London School of Medicine and Dentistry, Wolfson Institute of Preventive Medicine, Queen Mary University of London, 55 Philpot Street, London, E1 2JH, UK; Tel: +44 207882 5949;

Fax: +44 207377 7237; E-mail:d.przulj@qmul.ac.uk subjectively rewarding than smoking, even when the dose of nicotine was matched to the dose inhaled from the cigarette [5-7].

Such observations and theories raise the possibility that smoking cessation treatments could perhaps be enhanced if both nicotine and sensorimotor aspects of smoking were addressed. The nicotine inhalator is currently the only licensed smoking cessation medication which attempts to address both these factors $[8,9]$. However it remains a poor substitute for smoking. The inhalator needs to be puffed intensely over 20 minutes to provide appreciable nicotine levels and compliance with its recommended use is low [10]. It is thus possible that whatever gains the inhalator may provide in terms of behavioural replacement are cancelled by its limited nicotine delivery. The inhalator also provides only limited sensory replacement in that its taste, smell, airway stimulation etc. do not resemble cigarette effects closely.

A growing body of research exists investigating the efficacy of several other sensorimotor replacement products in alleviating tobacco withdrawal symptoms and aiding cessation. These include flavoured non-nicotine inhalators and aerosols, de-nicotinised cigarettes, and more recently, electronic cigarettes. This review aims to evaluate the evidence examining whether these three products can help to reduce cravings to smoke and facilitate smoking cessation. Tables 1 and 2 provide a summary of the effects of sensorimotor replacement on craving and smoking cessation, respectively.

\section{NON-NICOTINE INHALATORS AND AEROSOLS}

The main body of research in this area originates from Jed Rose and his group, currently at the Duke Centre for Nicotine and Smoking Cessation Research. Their early studies focused on three sensory replacement products; the citric acid aerosol, black pepper extract inhalator, and 
ascorbic acid aerosol, were chosen because of their ability to mimic the throat 'scratch' delivered by tobacco smoke [11].

The citric acid aerosol significantly reduced craving compared to controlled puffs of air [12], to a placebo aerosol which was unflavoured $[13,14]$, and produced effects comparable to a low nicotine and tar cigarette [12]. Any craving relief from the citric acid aerosol may however be shortlived; Levin et al. found significant craving reduction in the morning, compared to the placebo group, but no differences between groups at later time points [13]. Additionally, Behm et al. found a significant difference between the citric acid group and placebo controls on the first day of abstinence, but equivalent craving ratings from day 5 of abstinence [14]. In a smoking cessation trial comparing a combination of the citric acid aerosol with a nicotine patch and a placebo aerosol also in combination with the patch [15], relief from craving on the quit day was significantly higher in the citric acid group; however no differences were found on the Shiffman-Jarvik withdrawal questionnaire [16]. Two randomised, placebocontrolled smoking cessation trials have been conducted with the citric acid aerosol. The first $(\mathrm{N}=74)$ examined the efficacy of the citric acid aerosol alone, vs a placebo aerosol [14]. There was a significantly higher point prevalence abstinence rate in the citric acid group (20\% abstinent vs $0 \%$ of controls) at day 19 post-quit, as verified with exhaled carbon monoxide $(\mathrm{CO})$ readings, but only in participants with high baseline $\mathrm{CO}$. An analysis of overall abstinence rates was not reported. In the second trial $(\mathrm{N}=100)$, nicotine patches were used in combination with a citric acid inhalator or placebo for 10 weeks [15]. Ten-week CO-validated continuous abstinence rates were significantly higher in the citric acid group than placebo (19.5\% vs 6.8\%). However, when adjusted for baseline differences in participant characteristics (e.g. number of cigarettes smoked per day, number of years smoked, $\mathrm{CO}$ ) this effect was marginal ( $p=$ $0.06)$. By 24 weeks, nearly all participants had relapsed and there was no difference between the two groups.

Two trials studied an ascorbic acid aerosol in craving relief and smoking cessation [17]. In study $1(\mathrm{~N}=63)$, greater levels of craving were reported one week post-cessation in those using the aerosol compared to controls using nothing. Three weeks post-cessation, this effect was marginal. COvalidated point prevalence abstinence rates were significantly higher in the ascorbic acid group at days 3 and 22 post-cessation and marginally higher at one week. Abstinence rates by the 6 and 12 week follow ups fell below $20 \%$, with no differences between conditions. In study 2 , two different types of ascorbic acid aerosols were compared (fine $v s$ coarse particles). Since no control group was included, the findings are difficult to interpret.

One study investigated the use of a black pepper inhalator [18]. During a three-hour session following overnight abstinence, where participants used their allocated inhalators ad-libitum whilst remaining abstinent, the black pepper inhalator decreased craving to a greater extent than an inhalator with no cartridge, and a menthol flavoured inhalator. It could be argued that these results may be due to the distraction caused by the irritating effects of black pepper, although participants reported liking the black pepper and menthol inhalators more than the placebo, and airway sensory effects were only rated stronger for the black pepper in the chest, with no differences between inhalators in other areas.

No further work has been conducted with the citric, ascorbic and black pepper inhalators due to extensive regulatory requirements. However, a recent smoking cessation trial $(\mathrm{N}=120)$ has investigated the use of a tobacco flavoured nicotine-free inhalator in combination with pharmacological (nicotine patch plus bupropion) and behavioural treatment [19]. The inhalator had no effect on abstinence rates at 4 or 24 weeks overall. However, there was a significant effect of the inhalator in smokers who reported high levels of 'behavioural dependence' at baseline, measured by the Glover-Nilsson Smoking Behaviour Questionnaire [20]. This post-hoc finding requires a replication.

In summary, flavoured non-nicotine inhalators used alone or in combination with the nicotine patch may alleviate craving to some extent compared with placebo, but the effects are likely to be short-lived. Theories of learning would predict that the removal of the unconditioned reinforcer (nicotine) would gradually weaken any effects of conditioned reinforcers (sensorimotor stimuli) until the reaction to them extinguishes altogether. However, even a short-term reduction of urges to smoke may facilitate the initial abstinence, which may in turn affect continuous cessation long-term. It is unfortunate that further work with the citric, ascorbic acid and black pepper inhalators had to be halted, but recently two other promising approaches have emerged.

\section{DE-NICOTINISED CIGARETTES}

De-nicotinised cigarettes (DNCs) contain tobacco with almost all the nicotine removed (machine yield $<0.1 \mathrm{mg}$ nicotine). DNCs differ from 'light' and 'ultra-light' cigarettes which also register low levels of nicotine when assessed mechanically, but allow smokers to obtain standard doses of nicotine by blocking ventilation holes and puffing more intensively. This is possible because the tobacco in such cigarettes still contains nicotine levels similar to high nicotine yield cigarettes [21]. DNCs provide negligible levels of nicotine to smokers even with intensive puffing. Otherwise however, they deliver all other chemicals normally present in cigarette smoke and provide a virtually complete behavioural and sensory replacement for cigarettes. If sensorimotor replacement helps smokers over the initial withdrawal period, DNCs should be more helpful than other sensorimotor replacement products. Since DNCs deliver most of the chemicals found in conventional cigarettes, including those assumed to enhance nicotine effects, they may also potentiate the therapeutic effects of NRT.

The majority of studies with DNCs have examined the acute effects of DNCs after overnight abstinence. Measures of craving have varied across studies, from single item questions to more comprehensive questionnaires such as the Questionnaire of Smoking Urges (QSU) [22] which measures desires or intentions to smoke (Factor 1) and anticipation of relief from withdrawal and negative affect (Factor 2). Most studies have compared DNCs to conventional cigarettes. 
Table 1. Summary of the Effects of Sensorimotor Replacement Products on Craving

\begin{tabular}{|c|c|c|c|c|}
\hline Author & Product & $\mathbf{N}$ & Design & Findings \\
\hline $\begin{array}{l}\text { Rose \& Hickman } \\
\text { (1987) }\end{array}$ & $\begin{array}{l}\text { Citric acid } \\
\text { aerosol }\end{array}$ & 15 & $\begin{array}{l}\text { Within-subjects. } \\
\text { Single-blind. } \\
\text { Pre-study abstinence: } 1 \text { hour. }\end{array}$ & $\begin{array}{l}\text { Sig. less craving } v s \text { inhalations of air. } \\
\text { Comparable levels } v s \text { 'light' cigarette. } \\
\text { Sig. more craving } v s \text { OB* }^{*}\end{array}$ \\
\hline $\begin{array}{l}\text { Levin et al. (1990), } \\
\text { exp. } 2 .\end{array}$ & $\begin{array}{l}\text { Citric acid } \\
\text { aerosol }\end{array}$ & 11 & $\begin{array}{l}\text { Between-subjects, randomised. } \\
\text { Single-blind. } \\
\text { Pre-study abstinence: unknown. }\end{array}$ & Sig. less craving $v s$ placebo, in the morning only. \\
\hline Behm et al. (1993) & $\begin{array}{l}\text { Citric acid } \\
\text { aerosol }\end{array}$ & 74 & $\begin{array}{l}\text { Randomised smoking cessation trial. } \\
\text { Double-blind. }\end{array}$ & Sig. less craving $v s$ placebo on day 1 of abstinence only. \\
\hline Westman et al. (1995) & $\begin{array}{l}\text { Citric acid } \\
\text { aerosol }+ \\
\text { nicotine } \\
\text { patch }\end{array}$ & 100 & $\begin{array}{l}\text { Randomised smoking cessation trial. } \\
\text { Double-blind. }\end{array}$ & $\begin{array}{l}\text { "Craving relief": } \\
\text { Sig. greater } v s \text { placebo on day } 1 \text { of abstinence. } \\
\text { Shiffman-Jarvik craving subscale: } \\
\text { Comparable levels of craving } v s \text { placebo. }\end{array}$ \\
\hline $\begin{array}{l}\text { Levin et al. (1993), } \\
\text { Study } 1 .\end{array}$ & $\begin{array}{l}\text { Ascorbic } \\
\text { acid }\end{array}$ & 63 & Randomised smoking cessation trial. & $\begin{array}{l}\text { Sig. greater craving } v s \text { controls on day } 8 \text { of abstinence. } \\
\text { Comparable levels thereafter. }\end{array}$ \\
\hline Rose \&Behm (1994) & $\begin{array}{l}\text { Black } \\
\text { pepper } \\
\text { inhalator }\end{array}$ & 48 & $\begin{array}{l}\text { Between- subjects, randomised. } \\
\text { Pre-study abstinence: overnight. }\end{array}$ & $\begin{array}{l}\text { Sig. greater reduction in craving } v s \text { placebo and menthol } \\
\text { flavoured inhalator. }\end{array}$ \\
\hline Hasenfratz et al. (1993) & DNCs & 12 & $\begin{array}{l}\text { Within-subjects. } \\
\text { Pre-study abstinence: overnight. }\end{array}$ & $\begin{array}{l}\text { Comparable levels of craving reduction } v s \text { 'light' } \\
\text { cigarettes. } \\
\text { Sig. greater reduction with OB. }\end{array}$ \\
\hline Rose et al. (1994) & DNCs & 12 & $\begin{array}{l}\text { Within-subjects. } \\
\text { Pre-study abstinence: overnight. }\end{array}$ & $\begin{array}{l}\text { Comparable levels of craving reduction vs nicotine } \\
\text { cigarette. }\end{array}$ \\
\hline Butchsky et al. (1995) & DNCs & 7 & $\begin{array}{l}\text { Within-subjects, randomised. } \\
\text { Pre-study abstinence: } 12 \text { hours. }\end{array}$ & $\begin{array}{l}\text { Comparable levels of craving reduction } v s \text { nicotine } \\
\text { cigarette. }\end{array}$ \\
\hline Baldinger et al. (1995) & DNCs & 12 & $\begin{array}{l}\text { Within-subjects. } \\
\text { Pre-study abstinence: overnight. }\end{array}$ & Comparable levels of craving $v s$ 'light' cigarettes and OB. \\
\hline Baldinger et al. (1995) & DNCs & 12 & $\begin{array}{l}\text { Within-subjects. } \\
\text { Pre-study abstinence: overnight. }\end{array}$ & $\begin{array}{l}\text { Sig. less craving } v s \text { no-intervention condition. } \\
\text { Comparable levels of craving } v s \text { OB. }\end{array}$ \\
\hline Lane et al. (1995) & DNCs & 18 & $\begin{array}{l}\text { Within-subjects. } \\
\text { Single-blind. } \\
\text { Pre-study abstinence: overnight. }\end{array}$ & $\begin{array}{l}\text { Sig. less craving } v s \text { no-intervention condition. } \\
\text { Sig. more craving } v s \text { OB }\end{array}$ \\
\hline Westman et al. (1996) & DNCs & 6 & $\begin{array}{l}\text { Within-subjects, randomised. } \\
\text { Pre-study abstinence: overnight. }\end{array}$ & Comparable levels of craving $v s$ nicotine cigarette. \\
\hline Gross et al. (1997) & DNCs & 10 & $\begin{array}{l}\text { Within-subjects, randomised. } \\
\text { Single-blind. } \\
\text { Pre-study abstinence: none. }\end{array}$ & Comparable levels of craving $v s$ 'light' cigarettes and OB. \\
\hline Pickworth et al. (1999) & DNCs & 20 & $\begin{array}{l}\text { Within-subjects, randomised. } \\
\text { Double-blind. } \\
\text { Pre-study abstinence: overnight and } 3 \\
\text { hours. }\end{array}$ & $\begin{array}{l}\text { Comparable levels of craving reduction } v s \text { nicotine } \\
\text { cigarettes. } \\
\text { No main effect of abstinence length. }\end{array}$ \\
\hline Buchhalter et al. (2001) & DNCs & 32 & $\begin{array}{l}\text { Within-subjects. } \\
\text { Single-blind. } \\
\text { Pre-study abstinence: } 8 \text { hours. }\end{array}$ & $\begin{array}{l}\text { QSU: } \\
\text { Sig. less craving vs Accord device. } \\
\text { Comparable levels vs OB. } \\
\text { "Craving a cigarettes/nicotine": } \\
\text { Comparable levels of craving } v s \text { Accord device. }\end{array}$ \\
\hline Breland et al. (2002) & DNCs & 20 & $\begin{array}{l}\text { Within-subjects. } \\
\text { Single-blind. } \\
\text { Pre-study abstinence: overnight. }\end{array}$ & $\begin{array}{l}\text { Sig. less craving } v s \text { Accord device. } \\
\text { Comparable levels of craving vs Eclipse device and OB. }\end{array}$ \\
\hline Rose et al. (2003) & DNCs & 18 & $\begin{array}{l}\text { Within-subjects. } \\
\text { Pre-study abstinence: overnight. }\end{array}$ & $\begin{array}{l}\text { Sig. greater craving reduction } v s \text { saline control condition. } \\
\text { Sig less craving reduction } v s \text { OB. }\end{array}$ \\
\hline
\end{tabular}


(Table 1) contd.....

\begin{tabular}{|c|c|c|c|c|}
\hline Dallery et al. (2003) & DNCs & 15 & $\begin{array}{l}\text { Within-subjects. } \\
\text { Pre-study abstinence: } 30 \text { minutes. }\end{array}$ & Comparable reductions in craving $v s$ nicotine cigarettes. \\
\hline Buchhalter et al. (2005) & DNCs & 32 & $\begin{array}{l}\text { Within-subjects. } \\
\text { Double-blind. } \\
\text { Pre-study abstinence: N/A, } \\
\text { participants remained abstinent over } 4 \\
\text { days. }\end{array}$ & $\begin{array}{l}\text { "Urges to smoke": } \\
\text { No increase in craving over } 4 \text { days in DNC and nicotine } \\
\text { cigarette condition, but sig. increase in no-intervention } \\
\text { condition. } \\
\text { "Craving a cigarette ": } \\
\text { Sig. increases on most days vs baseline, in both DNC and } \\
\text { no-intervention conditions, but no changes in craving in } \\
\text { nicotine cigarette condition. } \\
\text { QSU-Factor 1: } \\
\text { No increase in craving over } 4 \text { days in DNC and nicotine } \\
\text { cigarette condition, but sig. increase in no-intervention } \\
\text { condition. } \\
\text { QSU-Factor 2: } \\
\text { Sig. increases on most days } v s \text { baseline, in both DNC and } \\
\text { no-intervention conditions, but no changes in craving in } \\
\text { nicotine cigarette condition. }\end{array}$ \\
\hline Eid et al. (2005) & DNCs & 8 & $\begin{array}{l}\text { Within-subjects, randomised. } \\
\text { Pre-study abstinence: none. }\end{array}$ & Comparable reductions in craving $v s$ nicotine cigarette. \\
\hline Juliano et al. (2006) & DNCs & 60 & $\begin{array}{l}\text { Between-subjects, randomised. } \\
\text { Double-blind. } \\
\text { Pre-study abstinence: } 4 \text { days. }\end{array}$ & $\begin{array}{l}\text { Sig. greater reduction in craving } v s \text { no-intervention control } \\
\text { group. } \\
\text { Comparable levels of craving reduction } v s \text { nicotine } \\
\text { cigarette. }\end{array}$ \\
\hline Rose et al. (2006) & $\begin{array}{l}\text { DNCs }+ \\
\text { nicotine } \\
\text { patch } \\
\text { (before } \\
\text { TQD). }\end{array}$ & 96 & $\begin{array}{l}\text { Randomised smoking cessation trial. } \\
\text { DNCs un-blinded. }\end{array}$ & $\begin{array}{l}\text { During treatment, and } 1 \text { week post-quit day, sig. less } \\
\text { craving in the DNC + placebo patch group vs OB + placebo } \\
\text { patch group. } \\
\text { Comparable levels at } 4 \text { weeks post-quit. }\end{array}$ \\
\hline $\begin{array}{l}\text { Rezaishiraz et al. } \\
\text { (2007) }\end{array}$ & $\begin{array}{l}\text { DNCs }+ \\
\text { nicotine } \\
\text { patch } \\
\text { (before } \\
\text { TQD) }\end{array}$ & 98 & $\begin{array}{l}\text { Randomised smoking cessation trial. } \\
\text { Un-blinded. }\end{array}$ & $\begin{array}{l}\text { During treatment and } 2 \text { weeks post-quit day, sig. less } \\
\text { craving } v s \text { 'light' cigarette group. }\end{array}$ \\
\hline Brody et al. (2009) & DNCs & 62 & $\begin{array}{l}\text { Between-subjects, randomisation } \\
\text { unclear. } \\
\text { Double-blind. } \\
\text { Pre-study abstinence: } 3 \text { hours. }\end{array}$ & Comparable reductions in craving $v s \mathrm{OB}$. \\
\hline Barrett (2010) & DNCs & 22 & $\begin{array}{l}\text { Within-subjects. } \\
\text { Double-blind. } \\
\text { Pre-study abstinence: } 12 \text { hours. }\end{array}$ & $\begin{array}{l}\text { QSU-Factor 1: } \\
\text { Sig. less craving vs nicotine and placebo inhalator. } \\
\text { Comparable levels of craving vs nicotine cigarette. } \\
\text { QSU-Factor 2: } \\
\text { Sig. less craving vs placebo inhalator and comparable } \\
\text { levels } v \text { nicotine inhalator. } \\
\text { Comparable levels of craving } v s \text { nicotine cigarette. }\end{array}$ \\
\hline Cobb et al. (2010) & DNCs & 28 & $\begin{array}{l}\text { Within-subjects. } \\
\text { Single-blind. } \\
\text { Pre-study abstinence: overnight. }\end{array}$ & $\begin{array}{l}\text { Sig. less craving } v s \text { baseline measures. } \\
\text { Comparisons between DNCs and other products not } \\
\text { reported, but DNCs showed similar patterns of results to } \\
\text { OB condition. }\end{array}$ \\
\hline Hatsukami et al. (2010) & DNCs & 165 & $\begin{array}{l}\text { Randomised smoking cessation trial. } \\
\text { Single-blind. }\end{array}$ & $\begin{array}{l}\text { No changes in craving } 1 \text { week post-quit. } \\
\text { Craving increased sig. following cessation of smoking } \\
\text { either DNCs or nicotine cigarettes } v \text { s previous week, but no } \\
\text { increases in lozenge group. }\end{array}$ \\
\hline
\end{tabular}




\begin{tabular}{|c|c|c|c|c|}
\hline Author & Product & $\mathbf{N}$ & Design & Findings \\
\hline Perkins et al. (2010) & DNCs & 104 & $\begin{array}{l}\text { Between-subjects, randomised. } \\
\text { Single-blind. } \\
\text { Pre-study abstinence: overnight. }\end{array}$ & $\begin{array}{l}\text { Sig. greater reduction in craving } v s \text { no-intervention control } \\
\text { group. } \\
\text { Comparable craving reduction } v s \text { nicotine cigarette group. }\end{array}$ \\
\hline Rose et al. (2010) & DNCs & 16 & $\begin{array}{l}\text { Within-subjects. } \\
\text { Single-blind. } \\
\text { Pre-study abstinence: overnight. }\end{array}$ & Sig. less craving $v s$ sham smoking. \\
\hline Walker et al. (2011) & $\begin{array}{l}\text { DNCs }+ \\
\text { NRT }\end{array}$ & 1410 & $\begin{array}{l}\text { Randomised smoking cessation trial. } \\
\text { Single-blind. }\end{array}$ & $\begin{array}{l}\text { Comparable levels of craving over } 6 \text { weeks post-quit } v s \\
\text { NRT alone. }\end{array}$ \\
\hline Bullen et al. (2010) & EC & 40 & $\begin{array}{l}\text { Within-subjects. } \\
\text { Double-blind. } \\
\text { Pre-study abstinence: overnight. }\end{array}$ & $\begin{array}{l}\text { Sig. greater reduction in craving with nicotine EC } v s \\
\text { nicotine-free EC, only after } 25 \text { minutes post-product use. } \\
\text { Comparable levels of craving } v s \text { nicotine inhalator. } \\
\text { Sig. less craving reduction } v s \text { OB. }\end{array}$ \\
\hline Eissenberg (2010) & $\mathrm{EC}$ & 16 & $\begin{array}{l}\text { Within-subjects. } \\
\text { Pre-study abstinence: } 12 \text { hours. }\end{array}$ & $\begin{array}{l}\text { Comparable levels of craving } v s \text { baseline and sham } \\
\text { smoking at most time points. } \\
\text { Sig. less craving reduction } v s \text { OB. }\end{array}$ \\
\hline Vansickel et al. (2010) & EC & 32 & $\begin{array}{l}\text { Within-subjects. } \\
\text { Pre-study abstinence: } 12 \text { hours. }\end{array}$ & $\begin{array}{l}\text { Sig. less craving } v s \text { baseline and sham smoking at some } \\
\text { time points. } \\
\text { Sig. less craving reduction } v s \text { OB. }\end{array}$ \\
\hline
\end{tabular}

Not surprisingly, data pooled from 9 studies showed DNCs' effects to be weaker [23]. Mean cigarette reward ratings (satisfaction and craving reduction) were $4.05(\mathrm{SD}=0.15)$ for nicotine cigarettes, and $3.36(\mathrm{SD}=0.14)$ for DNCs $(p$ $<.001)$. The size of this effect is unknown, and regression analyses revealed significant individual differences, in that males and more dependent smokers reported more similar ratings between the two types of cigarette. A fair number of studies have however found similar craving suppression to nicotine containing cigarettes [7, 24-42].

Of more interest in our context are the effects of DNCs compared to no intervention and to alternative craving reduction techniques. There is good evidence that DNCs alleviate craving acutely compared to no intervention [37, $41,43]$. DNCs were also more effective than puffing on an unlit cigarette or taking in puffs of air $[40,44]$. In one study, DNCs smoked alongside intravenous delivery of saline, were more effective at reducing cravings compared to saline alone [6]. DNCs also reduced urges to smoke compared to baseline over two hours (following overnight abstinence), as did snus (non-combustible tobacco product), whilst nicotine lozenge and a compressed tobacco tablet had no significant effect [40]. The report however does not indicate whether there were any differences between the DNCs and noncombustible products. DNCs have also been compared to potential reduced exposure products such as Accord and Eclipse, which heat tobacco. Although both Accord and Eclipse were able to reduce cravings relative to baseline, DNCs were more effective than the Accord device [31, 32], and equivalent to the Eclipse [31]. Finally, DNCs were more effective than a placebo and nicotine inhalator in reducing intentions to smoke (Factor 1, QSU) and equal to a nicotine inhalator in alleviating withdrawal/negative affect (Factor 2, QSU) over 2 hours following at least 12 hours of abstinence [42].

Three studies have investigated the effects of DNCs over longer periods of time. Over 24 hours of abstinence, craving was significantly lower with DNCs than with no intervention [28] and this was also observed on some, though not all, measures of craving over 4 days of abstinence [36]. However, in a well-controlled study which hospitalised volunteers for 13 days, participants in the DNC and notreatment conditions did not differ in ratings of craving over 11 days of abstinence [38].

In three randomised controlled trials DNCs have been used for several weeks prior to the quit date but not after it. In a complicated and underpowered trial which randomised 96 participants into 6 conditions, DNCs unexpectedly generated significantly greater reduction in craving compared to own brand smoking over two weeks of use, as well as on the quit day and one week post-quit. There was no effect on abstinence rates at 1 and 6 months [45]. In the second trial, 98 participants were given nicotine patches and were randomised to smoke DNCs or low-nicotine cigarettes for 2 weeks before quitting. Craving was significantly lower in the DNC group, both before the quit day and at 2 weeks post-quit, but self-reported abstinence rates at 3 and 6 months did not differ [46]. Finally, in a larger trial $(\mathrm{N}=346)$ participants used cigarettes with gradually reduced nicotine content over 6 weeks until DNCs were smoked in the final two weeks. Participants also used a placebo or nicotine patch, before and after the quit day. A control group smoked normal cigarettes during the pre-quit period and used a nicotine patch following the quit day. All groups were asked to stop smoking all cigarettes after the target quit date. 
Table 2. Summary of the Effects of Sensorimotor Products on Smoking Cessation

\begin{tabular}{|c|c|c|c|c|}
\hline Author & Design & $\mathbf{N}$ & Intervention & Abstinence Rates \\
\hline $\begin{array}{l}\text { Behm et al. } \\
\text { (1993) }\end{array}$ & $\begin{array}{l}\text { Randomised. } \\
\text { Double-blind. }\end{array}$ & 74 & $\begin{array}{l}\text { Citric acid aerosol. } \\
\text { Placebo aerosol. } \\
\text { All received group support. }\end{array}$ & $\begin{array}{l}\text { PP*, day 19: } \\
\text { High CO** group: } \\
20 \% \text { citric acid } v s 0 \% \text { placebo }(p<0.05) \text {. } \\
\text { Low CO group: } \\
20 \% \text { citric acid } v s 25 \% \text { placebo }(n s)\end{array}$ \\
\hline $\begin{array}{l}\text { Westman et } \\
\text { al. (1995) }\end{array}$ & $\begin{array}{l}\text { Randomised. } \\
\text { Double-blind. }\end{array}$ & 100 & $\begin{array}{l}\text { Citric acid aerosol + nicotine patch. } \\
\text { Placebo aerosol + nicotine patch. } \\
\text { All received brief individual support. }\end{array}$ & $\begin{array}{l}\frac{\text { Continuous, } 10 \text { weeks (primary outcome): }}{19.5 \% \text { citric acid } v s 6.8 \% \text { placebo }(p<0.05} \text {, adjusted } p=0.06 \text { ). } \\
\frac{\text { Continuous, } 24 \text { weeks: }}{0 \% \text { citric acid, } v s 5.1 \% \text { placebo, }(n s) \text {. }} \\
\left.\frac{\text { Continuous, } 6 \text { weeks: }}{34.1 \% \text { citric acid } v s 1} 1.9 \% \text { placebo (adjusted, } p<0.01\right) \text {. } \\
\frac{\text { Continuous, } 4 \text { weeks: }}{36.6 \% \text { citric acid } v s 18.6 \% \text { placebo (adjusted } p<0.05) \text {. }}\end{array}$ \\
\hline $\begin{array}{l}\text { Levin et al. } \\
\text { (1993), Study } \\
1\end{array}$ & Randomised. & 63 & $\begin{array}{l}\text { Ascorbic acid + group support. } \\
\text { Group support only. }\end{array}$ & $\begin{array}{l}\frac{\text { PP, day 3: }}{\sim 84 \% \text { ascorbic acid } v s} 60 \% \text { controls }(p=0.05) \\
\frac{\text { PP, day } 8:}{\sim 73 \% \text { ascorbic acid } v s ~} \sim 52 \% \text { controls }(p=0.09) \text {. } \\
\frac{\text { PP, day } 22:}{\sim 58 \% \text { ascorbic acid } v s \sim 22 \% \text { controls }(p<0.01)} \\
\frac{\text { PP, day } 43:}{20 \%, \text { both groups. }} \\
\frac{\text { PP, day } 85:}{\sim 16 \%, \text { both groups. }}\end{array}$ \\
\hline $\begin{array}{l}\text { Caponnetto et } \\
\text { al. (2011) }\end{array}$ & Randomised. & 120 & $\begin{array}{l}\text { Nicotine free, flavoured inhalator }+ \\
\text { pharmacological and behavioural } \\
\text { support. } \\
\text { Pharmacological and behavioural } \\
\text { support only. }\end{array}$ & $\begin{array}{l}\text { PP, } 4 \text { weeks: } \\
38.3 \% \text { inhalator vs } 35 \% \text { controls }(n s) \\
\frac{\text { PP, } 24 \text { weeks: }}{33.3 \% \text { inhalator vs } 28.3 \% \text { controls }(n s) \text {. }}\end{array}$ \\
\hline $\begin{array}{l}\text { Rose et al. } \\
\text { (2006) }\end{array}$ & $\begin{array}{l}\text { Randomised. } \\
\text { Cigarettes- un- } \\
\text { blinded. } \\
\text { Patches- } \\
\text { double-blind. }\end{array}$ & 96 & $\begin{array}{l}2 \text { weeks before TQD***: } \\
\text { 1. DNC + nicotine patch or placebo. } \\
\text { 2. 'Light' cigarette + nicotine patch or } \\
\text { placebo. } \\
\text { 3. OB+ nicotine patch or placebo. } \\
\frac{\text { After TQD: }}{42 \mathrm{mg}, 21 \mathrm{mg} \text {, or placebo patch. }} \\
\text { All received Mecamylamine or } \\
\text { placebo, and brief support. }\end{array}$ & $\begin{array}{l}\text { Continuous, } 4 \text { weeks: } \\
\text { Sig. main effect of pre-cessation patch only }(\mathrm{p}<0.01) \text {. } \\
50 \% \text { DNC + patch } v s 23 \% \text { placebo. } \\
50 \% \text { 'light' cigarette }+ \text { patch } v s 33 \% \text { placebo. } \\
50 \% \text { OB + patch } v s 12 \% \text { placebo. }\end{array}$ \\
\hline $\begin{array}{l}\text { Rezaishirazet } \\
\text { al. (2007) }\end{array}$ & $\begin{array}{l}\text { Randomised. } \\
\text { Un-blinded. }\end{array}$ & 98 & $\begin{array}{l}\text { 2 weeks before TQD: } \\
\text { DNC + nicotine patch. } \\
\text { 'Light' cigarettes only. } \\
\text { After TQD: } \\
\text { All received nicotine patch and } \\
\text { behavioural support. }\end{array}$ & $\begin{array}{l}\text { PP, } 3 \text { months: } \\
43 \% \text { DNC + patch } v s 34 \% \text { control group }(n s) . \\
\frac{\text { PP, } 6 \text { months: }}{28 \% \text { DNC }+ \text { patch } v s ~} 21 \% \text { control group }(n s) .\end{array}$ \\
\hline $\begin{array}{l}\text { Becker et al. } \\
(2008)\end{array}$ & $\begin{array}{l}\text { Randomised. } \\
\text { Double-blind. }\end{array}$ & 346 & $\begin{array}{l}6 \text { weeks before TQD: } \\
\text { Quest } 1,2,3 \dagger(2 \text { weeks each) }+ \text { nicotine } \\
\text { patch in last } 2 \text { weeks or placebo patch. } \\
\text { Conventional cigarettes + placebo } \\
\text { patch last } 2 \text { weeks (control group). } \\
\text { After TQD: } \\
\text { DNC groups received nicotine or } \\
\text { placebo patch. } \\
\text { Control group received nicotine patch. } \\
\text { All received brief behavioural support. }\end{array}$ & $\begin{array}{l}\text { Continuous, } 4 \text { weeks: } \\
32.8 \% \text { DNC + patch } v s 21.9 \% \text { control group }(p<0.05) \text {. } \\
16.4 \% \text { DNC + placebo } v s 21.9 \% \text { control group }(n s)\end{array}$ \\
\hline
\end{tabular}


(Table 2) contd....

\begin{tabular}{|c|c|c|c|c|}
\hline Author & Design & $\mathbf{N}$ & Intervention & Abstinence Rates \\
\hline $\begin{array}{l}\text { Hatsukami } \\
\text { et al. }(2010)\end{array}$ & $\begin{array}{l}\text { Randomised. } \\
\text { Single-blind. }\end{array}$ & 165 & $\begin{array}{l}\text { DNCs. } \\
\text { 'Light' cigarettes. } \\
\text { Lozenges. } \\
\text { All received weekly brief support. }\end{array}$ & 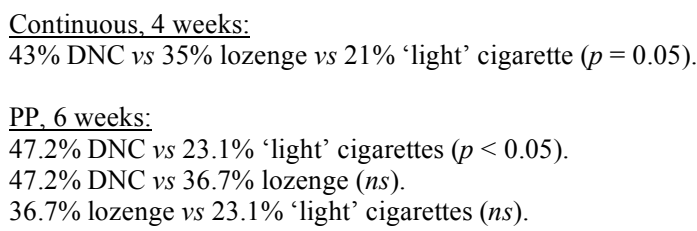 \\
\hline $\begin{array}{l}\text { Walker et al. } \\
\text { (2011) }\end{array}$ & $\begin{array}{l}\text { Randomised. } \\
\text { Single-blind. }\end{array}$ & 1,410 & $\begin{array}{l}\text { DNCs + NRT and behavioural support. } \\
\text { NRT and behavioural support only. }\end{array}$ & $\begin{array}{l}\text { PP, } 6 \text { months (primary outcome): } \\
33 \% \text { DNC vs } 28 \% \text { control group }(p<0.05, R R=1.18, C I= \\
1.01-1.39) \text {. } \\
\text { Continuous, } 6 \text { months: } \\
23 \% \text { DNC vs } 15 \% \text { Control group }(p<0.001, R R=1.50, C I= \\
1.20-1.87) \text {. }\end{array}$ \\
\hline
\end{tabular}

*PP: Point prevalence, ${ }^{* *} \mathrm{CO}$ : carbon monoxide, ${ }^{* * * T Q}$ QD: Target quit day.

$\dagger$ Quest 1,2,3: cigarettes with progressively reduced nicotine content. Quest 3 is de-nicotinised.

Craving measures were not reported in the study. Four week $\mathrm{CO}$-validated continuous abstinence rates were significantly higher in the DNC plus patch group vs controls (33\% vs $22 \%$, respectively), but the DNC plus placebo patch group did not differ in outcome relative to controls $(22 \%$ vs $16 \%$, respectively). Differences between the two DNC groups were not examined. By 3 and 6 months no differences in abstinence rates were present [47].

Two studies have examined the use of DNCs following the quit day. In one of them, DNCs were compared to nicotine lozenge and to low-nicotine cigarettes. Participants $(\mathrm{N}=165)$ used their assigned products ad-libitum for a period of 6 weeks starting on their target quit day. Following cessation of products, craving increased significantly for the two cigarette groups. Continuous $\mathrm{CO}$-validated quit rates at four weeks after discontinuation of the products (though use of lozenge was permitted) did not differ across conditions, but the trend favoured the DNC group (43\%, 35\%, and $21 \%$ for $\mathrm{DNC}$, lozenge, and low-nicotine cigarettes, respectively) [48]. In the second trial participants $(\mathrm{N}=1,410)$ were randomised to either standard care (NRT and behavioural support for 8 weeks) or standard care alongside DNC use for a period of 6 weeks after the quit day. There were no differences between groups in urges to smoke over 6 weeks. Abstinence rates were higher in the DNC group at all follow up points up to 6 months $(23 \%$ vs $15 \%$, p $<0.001)$. Abstinence however was not biochemically verified at any time point.

In summary, the existing evidence suggests that DNCs can alleviate craving acutely, and in some cases over longer periods of abstinence. DNCs have little effect on smoking cessation if used prior to quitting. However, they may provide some help if used alongside NRT following the quit day. There are some encouraging findings but the evidence is not conclusive and further trials with DNCs in combination with current smoking cessation treatments (NRT, varenicline) are needed.

${ }^{1}$ Walker N, Howe C, Bullen C et al. Can the use of nicotine free cigarettes as an adjunct to usual NRT-based cessation practice help people quit smoking? Findings from a randomised trial: 2011: Poster presentation at the $13^{\text {th }}$ Annual Meeting of the SRNT- Europe; 2011 Sep 8-11; Antalya, Turkey.

\section{ELECTRONIC CIGARETTES}

The final product which may be of use as a sensorimotor replacement for smoking is the electronic cigarette (EC). ECs are tobacco free, battery powered devices. They typically resemble conventional cigarettes in appearance and with each puff a visible vapour or mist is created which resembles smoke. Importantly, this provides sensorimotor stimuli fairly close to smoking (e.g. throat scratch, inhaling/exhaling). Cartridges for ECs can be purchased with differing levels of nicotine, including nicotine-free, and in a variety of different flavours.

ECs have been commercially available since 2004, and five studies to date have been published examining their efficacy in acute craving reduction. Two of these have compared two brands of ECs (NPRO and Hydro) with own brand cigarettes and a sham smoking control condition, after 12 hours of abstinence [49, 50]. As expected, own brand cigarettes were found to reduce craving to a greater extent than ECs. ECs were reported to reduce cravings relative to baseline and sham smoking at some time points [50], but in the other trial they showed little impact on baseline craving or difference from sham smoking [49]. It should be noted that both of these early studies allowed only 10 puffs of the EC and no increases in plasma nicotine levels were observed. Any effects in these two studies were thus due to sensorimotor stimulation rather than nicotine.

In a direct comparison of a nicotine and placebo EC [51], significantly greater reductions in craving over one hour were evident with the nicotine EC. However the placebo EC also reduced craving initially, with the differences between the two arms only becoming apparent at 25 minutes post product use and onwards. This study also compared the ECs to own brand cigarettes and a nicotine inhalator. As before, own brand cigarettes reduced craving to a greater extent than all other products, but no differences were found between the ECs and inhalator. A further two studies have been published recently, one examining EC effects with experienced users [52] and another with naïve EC users [53]. However in both of these studies no control group was included.

The efficacy of ECs outside of controlled laboratory settings and over longer periods of time is as yet unknown. Several internet surveys of EC users have reported that the majority of respondents have successfully replaced their 
usual cigarettes with ECs [54-57]. Additionally, in one study, participants who were not seeking to quit were given ECs to use ad-libitum for 6 months. At 6 months, 22.5\% had quit smoking [58]. Clinical trials are now needed to investigate EC efficacy in smoking reduction and cessation.

\section{DISCUSSION}

This review has summarised the current evidence on the effects of three sensorimotor replacement products (nonnicotine inhalators and aerosols; de-nicotinised cigarettes; and electronic cigarettes) in craving reduction and smoking cessation. In summary, flavoured non-nicotine inhalators used alone or in combination with the nicotine patch may alleviate craving to some extent compared with placebo, but the effects are fairly small and short-lived. DNCs alleviate craving in the short term at least, and may be a useful tool for smoking cessation when used after the quit day, and in combination with NRT. The evidence base on ECs is currently limited, but these early studies suggest they may be a promising tool for withdrawal relief and smoking cessation.

DNCs appear to lend the most support for the sensorimotor replacement hypothesis, with a number of studies showing equivalent acute craving suppression to even conventional nicotine cigarettes. DNCs provide an almost full sensory and behavioural replacement to smoking, compared to for example nicotine-free inhalators, which provide fewer conditioned sensorimotor stimuli. The one study which has compared DNCs to a nicotine-free inhalator indeed found DNCs to be more effective in alleviating craving [42]. Furthermore, other constituents of tobacco smoke present in DNCs may have direct pharmacological effects or enhance effects of nicotine from NRT [2], adding to the potential efficacy of DNCs as a supplement to existing pharmacological treatments.

There are however several concerns which may be slowing down work in this area. In theory, DNCs may prevent patients' habituation to life without cigarettes, and cessation of their use may represent a loss of a coping tool and precipitate withdrawal discomfort and relapse. In one study, participants exposed to DNCs following 4 days of abstinence relapsed back to smoking faster than those in the no-lapse condition [37]; but the sample consisted of smokers not wanting to quit. It is unlikely that users can become dependent on DNCs in the absence of the primary reinforcer. Any conditioned effects should dissipate over time, leading to a reduction in the number of DNCs smoked. In the two studies which examined DNC use post quit day, there was indeed a significant reduction in DNC use over time. These issues however require further empirical examination. Another concern relates to the fact that DNCs are as harmful to health as conventional cigarettes, although using them for a few weeks instead of conventional cigarettes to facilitate cessation of all tobacco use should be acceptable. Regarding ECs, these deliver sensorimotor stimuli closer to smoking than inhalators and aerosols though not as close as DNCs. However, they also deliver nicotine. Survey data have shown that some smokers have successfully switched from conventional cigarettes to ECs, suggesting that ECs may have the potential to compete with cigarettes as a consumer product. Questions remain as to whether ECs should be seen in the same light as for instance consumer products containing caffeine (e.g. teabags, soft drinks), or whether they should be submitted to stricter regulation in the absence of any evidence of harm so far. Some governing bodies have banned or restricted the marketing and sales of ECs, while others such as the UK allow them on the basis that these products are not marketed for therapeutic use. The popularity of ECs is growing however [59], and regulations will no doubt be reviewed in light of further research on safety and efficacy.

This review has identified several pointers for future research. Regarding methodological issues, the majority of existing studies used laboratory procedures following overnight/12 hours of abstinence. Few studies have used more ecologically valid designs and examined effects of these products over longer periods of time. Studies of smoking cessation have not always complied with the Russell Standard [60], for example, not validating selfreported abstinence biochemically or reporting continuous abstinence. Regarding research priorities, studies are needed on the effects of DNCs and ECs as self-standing interventions for smoking reduction and cessation; and, perhaps more urgently, studies are needed on the effects of adding DNCs and ECs to existing treatments. Well powered large studies will also allow for testing the hypothesis that there are subgroups of smokers particularly likely to benefit from sensorimotor replacement.

In conclusion, sensorimotor replacement alone, used without nicotine, is likely to relieve cravings only briefly and it is unlikely to have a substantial effect in smoking cessation. However, it may increase the effectiveness of smoking cessation medications. ECs, which combine sensorimotor replacement with nicotine delivered contingent on the sensorimotor input, seem by far the most promising of the three approaches.

\section{ACKNOWLEDGEMENT}

Declared none.

\section{CONFLICT OF INTEREST}

Declared none.

\section{REFERENCES}

[1] Stead LF, Perera R, Bullen C, Mant D, Lancaster T. Nicotine replacement therapy for smoking cessation. Cochrane Database Syst Rev 2008; CD000146(1). Available from: http://onlinelibrary. wiley.com/doi/10.1002/14651858.CD000146.pub3/full. [cited: $3^{\text {rd }}$ Oct 2011].

[2] Rose J. Nicotine and nonnicotine factors in cigarette addiction. Psychopharmacology 2006; 184: 274-85.

[3] Rose JE, Levin ED. Inter-relationships between conditioned and primary reinforcement in the maintenance of cigarette smoking. $\mathrm{Br}$ J Addict 1991; 86: 605-9.

[4] Rose JE, Tashkin DP, Ertle A, Zinser MC, Lafer R. Sensory blockade of smoking satisfaction. Pharmacol Biochem Behav 1985; 23: 289-93.

[5] Rose JE, Behm FM, Westman EC, Johnson M. Dissociating nicotine and nonnicotine components of cigarette smoking. Pharmacol Biochem Behav 2000; 67:71-81.

[6] Rose JE, Behm FM, Westman EC, Bates JE, Salley A. Pharmacologic and sensorimotor components of satiation in cigarette smoking. Pharmacol Biochem Behav 2003; 76: 243-50.

[7] Westman EC, Behm FM, Rose JE. Dissociating the nicotine and airway sensory effects of smoking. Pharmacol Biochem Behav 1996; 53: 309-15. 
[8] Hajek P, Jarvis MJ, Belcher M, Sutherland G, Feyerabend C. Effect of smoke-free cigarettes on $24 \mathrm{~h}$ cigarette withdrawal: a doubleblind placebo-controlled study. Psychopharmacology 1989; 97: 99102.

[9] Schneider NG, Olmstead R, Nilsson F, Mody FV, Franzon M, Doan K. Efficacy of a nicotine inhaler in smoking cessation: a double-blind, placebo-controlled trial. Addiction 1996; 91: 1293306.

[10] Hajek P, West R, Foulds J, Nilsson F, Burrows S, Meadow A. Randomized comparative trial of nicotine polacrilex, a transdermal patch, nasal spray, and an inhaler. Arch Intern Med 1999; 159: 2033-8.

[11] Westman EC, Behm FM, Rose JE. Airway sensory replacement as a treatment for smoking cessation. Drug Dev Res 1996; 38: 257-62.

[12] Rose JE, Hickman CS. Citric acid aerosol as a potential smoking cessation aid. Chest 1987; 92: 1005-8.

[13] Levin ED, Rose JE, Behm F. Development of a citric acid aerosol as a smoking cessation aid. Drug Alcohol Depend 1990; 25: 273-9.

[14] Behm FM, Schur C, Levin ED, Tashkin DP, Rose JE. Clinical evaluation of a citric acid inhaler for smoking cessation. Drug Alcohol Depend 1993; 31: 131-8.

[15] Westman EC, Behm FM, Rose JE. Airway sensory replacement combined with nicotine replacement for smoking cessation. Chest 1995; 107: 1358-64.

[16] Shiffman SM, Jarvik ME. Smoking withdrawal symptoms in two weeks of abstinence. Psychopharmacology 1976; 50: 35-9.

[17] Levin ED, Behm F, Carnahan E, LeClair R, Shipley R, Rose JE. Clinical trials using ascorbic acid aerosol to aid smoking cessation. Drug Alcohol Depend1993; 33: 211-23.

[18] Rose JE, Behm FM. Inhalation of vapor from black pepper extract reduces smoking withdrawal symptoms. Drug Alcohol Depend 1994; 34: 225-9.

[19] Caponnetto P, Cibella F, Mancuso S, Campagna D, Arcidiacono G, Polosa R. Effect of a nicotine free inhalator as part of a smoking cessation program. Eur Respir J 2011; 38: 1005-11.

[20] Glover ED, Nilsson F, Westin A, Glover PN, Laflin MT, Persson B. Developmental history of the Glover-Nilsson smoking behavioral questionnaire. Am J Health Behav 2005; 29: 443-55.

[21] Kozlowski LT, Mehta NY, Sweeney CT, et al. Filter ventilation and nicotine content of tobacco in cigarettes from Canada, the United Kingdom, and the United States. Tob Control 1998; 7: 36975.

[22] Tiffany ST, Drobes DJ. The development and initial validation of a questionnaire on smoking urges. Br J Addict 1991; 86:1467-76.

[23] Brauer LH, Behm FM, Lane JD, Westman EC, Perkins C, Rose JE. Individual differences in smoking reward from de-nicotinized cigarettes. Nicotine Tob Res 2001; 3: 101-9.

[24] Hasenfratz M, Baldinger B, Bättig K. Nicotine or tar titration in cigarette smoking behavior? Psychopharmacology 1993; 112: 2538 .

[25] Butschky MF, Bailey D, Henningfield JE, Pickworth WB. Smoking without nicotine delivery decreases withdrawal in 12-hour abstinent smokers. Pharmacol Biochem Behav1995; 50: 91-6.

[26] Rose JE, Behm FM, Westman EC, et al. Combined effects of nicotine and mecamylamine in attenuating smoking satisfaction. Exp Clin Psychopharmacol 1994; 2: 328-44.

[27] Baldinger B, Hasenfratz M, Bättig K. Switching to ultralow nicotine cigarettes: Effects of different tar yields and blocking of olfactory cues. Pharmacol Biochem Behav1995; 50: 233-9.

[28] Baldinger B, Hasenfratz M, Bättig K. Effects of smoking abstinence and nicotine abstinence on heart rate, activity and cigarette craving under field conditions. Hum Psychopharmacol Clin 1995; 10: 127-36.

[29] Gross J, Lee J, Stitzer ML. Nicotine-containing vs de-nicotinized cigarettes: effects on craving and withdrawal. Pharmacol Biochem Behav 1997; 57: 159-65.

[30] Pickworth WB, Nelson RA, Rohrer MS, Fant RV, Henningfield JE. Pharmacodynamic effects of new de-nicotinized cigarettes. Nicotine Tob Res 1999; 1: 357-64.

[31] Breland AB, Buchhalter AR, Evans SE, Eissenberg T. Evaluating acute effects of potential reduced-exposure products for smokers: clinical laboratory methodology. Nicotine Tob Res 2002; 4(Suppl. 2): S131-40.

[32] Buchhalter AR, Schrinel L, Eissenberg T. Withdrawal-suppressing effects of a novel smoking system: comparison with own brand, not own brand, and de-nicotinized cigarettes. Nicotine Tob Res 2001; 3: 111-8.

[33] Dallery J, Houtsmuller E, Pickworth W, Stitzer M. Effects of cigarette nicotine content and smoking pace on subsequent craving and smoking. Psychopharmacology 2003; 165: 172-80.

[34] Rose JE, Behm FM. Effects of low nicotine content cigarettes on smoke intake. Nicotine Tob Res 2004; 6: 309-19.

[35] Eid NC, Fant RV, Moolchan ET, Pickworth WB. Placebo cigarettes in a spaced smoking paradigm. Pharmacol Biochem Behav 2005; 81: 158-64

[36] Buchhalter AR, Acosta MC, Evans SE, Breland AB, Eissenberg T. Tobacco abstinence symptom suppression: the role played by the smoking-related stimuli that are delivered by denicotinized cigarettes. Addiction 2005; 100: 550-9.

[37] Juliano LM, Donny EC, Houtsmuller EJ, Stitzer ML. Experimental evidence for a causal relationship between smoking lapse and relapse. J Abnorm Psychol 2006; 115: 166-73.

[38] Donny EC, Houtsmuller E, Stitzer ML. Smoking in the absence of nicotine: behavioral, subjective and physiological effects over 11 days. Addiction 2007; 102: 324-34.

[39] Brody AL, Mandelkern MA, Olmstead RE, et al. Ventral striatal dopamine release in response to smoking a regular vs a denicotinized cigarette. Neuropsychopharmacology 2009; 34: 2829.

[40] Cobb CO, Weaver MF, Eissenberg T. Evaluating the acute effects of oral, non-combustible potential reduced exposure products marketed to smokers. Tob Control 2010; 19: 367-73.

[41] Perkins KA, Karelitz JL, Conklin CA, Sayette MA, Giedgowd GE. Acute negative affect relief from smoking depends on the affect situation and measure but not on nicotine. Biol Psychiatry 2010; 67: 707-14.

[42] Barrett SP. The effects of nicotine, denicotinized tobacco, and nicotine-containing tobacco on cigarette craving, withdrawal, and self-administration in male and female smokers. Behav Pharmacol 2010; 21: 144-52.

[43] Lane JD, Lefebvre JC, Rose JE, Keefe FJ. Effects of cigarette smoking on perception of thermal pain. Exp Clin Psychopharmacol1995; 3:140-7.

[44] Rose JE, Salley A, Behm FM, Bates JE, Westman EC. Reinforcing effects of nicotine and non-nicotine components of cigarette smoke. Psychopharmacology 2010; 210: 1-12.

[45] Rose JE, Behm FM, Westman EC, Kukovich P. Precessation treatment with nicotine skin patch facilitates smoking cessation. Nicotine Tob Res 2006; 8: 89-101.

[46] Rezaishiraz H, Hyland A, Mahoney MC, O'Connor RJ, Cummings KM. Treating smokers before the quit date: can nicotine patches and denicotinized cigarettes reduce cravings? Nicotine Tob Res 2007; 9: 1139-46.

[47] Becker KM, Rose JE, Albino AP. A randomized trial of nicotine replacement therapy in combination with reduced-nicotine cigarettes for smoking cessation. Nicotine Tob Res 2008; 10: 113948 .

[48] Hatsukami DK, Kotlyar M, Hertsgaard LA, et al. Reduced nicotine content cigarettes: effects on toxicant exposure, dependence and cessation. Addiction 2010; 105: 343-55.

[49] Eissenberg T. Electronic nicotine delivery devices: ineffective nicotine delivery and craving suppression after acute administration. Tob Control 2010; 19: 87-8.

[50] Vansickel AR, Cobb CO, Weaver MF, Eissenberg TE. A clinical laboratory model for evaluating the acute effects of electronic "cigarettes": nicotine delivery profile and cardiovascular and subjective effects. Cancer Epidemiol Biomarkers Prev 2010; 19: 1945-53.

[51] Bullen C, McRobbie H, Thornley S, Glover M, Lin R, Laugesen M. Effect of an electronic nicotine delivery device (e cigarette) on desire to smoke and withdrawal, user preferences and nicotine delivery: randomised cross-over trial. Tob Control 2010; 19: 98103.

[52] Vansickel AR, Eissenberg T. Electronic cigarettes: effective nicotine delivery after acute administration. Nicotine Tob Res 2012; Available from: http://ntr.oxfordjournals.org/content/early/ 2012/02/05/ntr.ntr316.abstract. [cited: $7^{\text {th }}$ Feb 2012].

[53] Vansickel AR, Weaver MF, Eissenberg T. Clinical laboratory assessment of the abuse liability of an electronic cigarette. Addiction 2012; Available from: http://onlinelibrary.wiley.com/do i/10.1111/j.1360-0443.2012.03791.x/abstract. [cited: $7^{\text {th }}$ Feb 2012]. 
[54] Etter JF, Bullen C. Electronic cigarette: users profile, utilization, satisfaction and perceived efficacy. Addiction 2011; 106: 2017-28.

[55] Etter JF. Electronic cigarettes: a survey of users. BMC Public Health 2010; 10: 231 .

[56] Foulds J, Veldheer S, Berg A. Electronic cigarettes (e-cigs): views of aficionados and clinical/public health perspectives. Int $\mathrm{J}$ Clin Pract 2011; 65: 1037-42.

[57] Heavner KK, Dunworth J, Bergen PL, Nissen CM, Phillips CV. Electronic cigarettes (e-cigarettes) as potential tobacco harm reduction products: results of an online survey of e-cigarette user. Available from: tobaccoharmreduction.org/wpapers/011.htm. [cited: $22^{\text {nd }}$ Oct 2010].
[58] Polosa R, Caponnetto P, Morjaria JB, Papale G, Campagna D, Russo C. Effect of an electronic nicotine delivery device (eCigarette) on smoking reduction and cessation: a prospective 6month pilot study. BMC Public Health 2011; 11: 786.

[59] Ayers JW, Ribisl KM, Brownstein JS. Tracking the rise in popularity of electronic nicotine delivery systems (electronic cigarettes) using search query surveillance. Am J Prev Med 2011; 40: 448-53

[60] West R, Hajek P, Stead L, Stapleton J. Outcome criteria in smoking cessation trials: proposal for a common standard. Addiction 2005; 100: 299-303.

(C) Przulj et al.; Licensee Bentham Open.

This is an open access article licensed under the terms of the Creative Commons Attribution Non-Commercial License (http://creativecommons.org/licenses/ by-nc/3.0/) which permits unrestricted, non-commercial use, distribution and reproduction in any medium, provided the work is properly cited. 